\title{
Tinnitus severity correlated with zinc, copper, risk factors: a large-scale case-control study
}

\author{
Jiangfeng Huang ${ }^{1}$, Jing He${ }^{1}$, Qingping Zhu ${ }^{1}$, Zhiwei Zheng ${ }^{1}$, Peiji Zeng ${ }^{1}$, Wei Liu ${ }^{1}$, Jing \\ $\mathrm{Gao}^{1}$, Hanjing Shangguan ${ }^{1}$, Xianyang Luo ${ }^{1}$, Chengfu Cai ${ }^{1}$, Dongyan Shen ${ }^{1}$, Jinxing Shen ${ }^{1}$, \\ Mingqiang Lin ${ }^{1}$, Dong Mei Tang ${ }^{2}$, Huawei $\mathrm{Li}^{2}$, Baochang $\mathrm{He}^{3}$, and Lin $\mathrm{Cai}^{3}$ \\ ${ }^{1}$ Xiamen University and Fujian Medical University Affiliated First Hospital \\ ${ }^{2}$ Fudan University Eye Ear Nose and Throat Hospital \\ ${ }^{3}$ Fujian Medical University
}

April 28, 2020

\begin{abstract}
Objectives This large-scale case-control study aimed to explore the trace elements $(\mathrm{Zn} \mathrm{\&} \mathrm{Cu})$ and risk factors associated with tinnitus severity (mild and moderate-to-severe tinnitus).

Methods The serum levels of $\mathrm{Zn} \& \mathrm{Cu}$ of participants were measured by inductively coupled plasma mass spectrometry (ICP-MS). The potential risk factors were analyzed by simple and multiple logistic regression analysis.

Results Compared mild tinnitus with moderate-to-severe tinnitus, the serum $\mathrm{Zn}$ of participants had a significant difference $(\mathrm{P}=0.05)$, and only the age variable displayed an evident difference in main clinical characteristics analysis table $(\mathrm{P}<0.05)$. Under a multivariable-adjusted analysis, the potential risk factors included hearing loss (AOR: 1.704, 95\% CI: 1.009-2.880), HADS-A (borderline abnormal, AOR: 2.876, 95\% CI: 1.248-6.625; abnormal, AOR: 12.149, 95\% CI: 2.722-54.218), AIS (slight sleep problems, AOR: 2.030, 95\% CI: 1.061-3.885; probable/definite insomnia, AOR: 6.955, 95\% CI: 3.669-13.185), ear-selfcleaning (<1 t/w, AOR: 2.117, 95\% CI: 1.178-3.805; 3-6 t/w, AOR: 2.462, 95\% CI: 1.081-5.607; [?]7 t/w, AOR: 2.472, 95\% CI: 1.041-5.868), tea consumption (AOR: 1.138, 95\% CI: 1.052-1.231) and sleep apnea (AOR: 1.805, 95\% CI: 1.036-3.145). Next, a stratified analysis was made on these risk factors, and the results showed that the low levels of Zn were significantly associated with tinnitus severity in hearing loss group —-both in "no" and "yes" subgroups ("no", AOR: 2.588, 95\% CI: 1.348-6.061; "yes", AOR: 4.213, 95\% CI: 1.106-8.430), and in HADS-A group—noly in "normal" subgroup (AOR: $2.928,95 \%$ CI: $1.790-6.984)$.
\end{abstract}

Conclusions Serum Zn deficiency and potential risk factors (including hearing loss, tea consumption, sleep apnea, anxiety, insomnia and ear-self-cleaning habit) were significantly correlated with tinnitus severity. Intervention with these risk factors could prevent the mild tinnitus from becoming moderate-to-severe tinnitus.

\section{Hosted file}

0421-_Document.doc available at https : //authorea.com/users/314775/articles/445209-tinnitus-severitycorrelated-with-zinc-copper-risk-factors-a-large-scale-case-control-study

\section{Hosted file}

0421-Table.docx available at https: //authorea.com/users/314775/articles/445209-tinnitus-severitycorrelated-with-zinc-copper-risk-factors-a-large-scale-case-control-study 

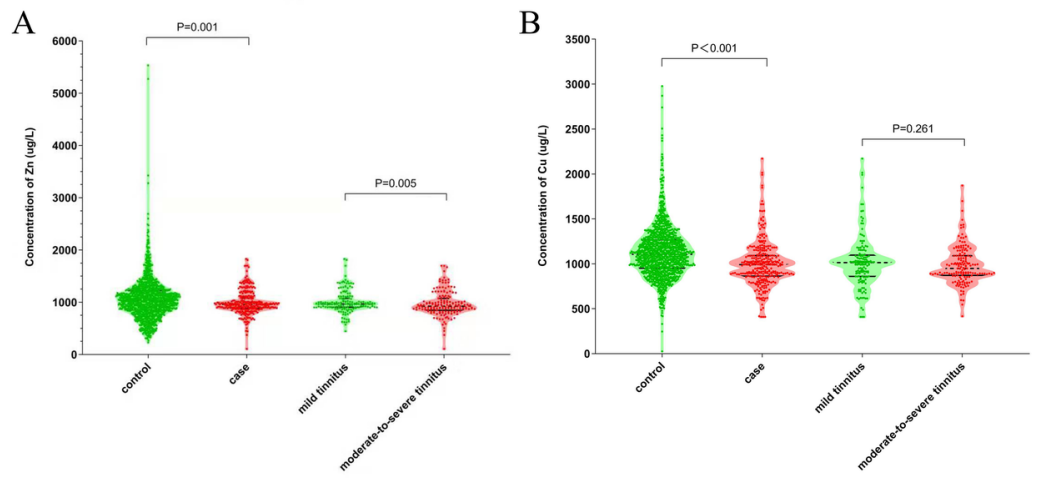